\title{
Overexpression of uncoupling protein 2 inhibits the high glucose-induced apoptosis of human umbilical vein endothelial cells
}

\author{
$\mathrm{YING} \mathrm{HE}^{1^{*}}, \mathrm{ZHOU} \mathrm{LUAN}^{2 *}, \mathrm{XUNAN} \mathrm{FU}^{1}$ and $\mathrm{XUN} \mathrm{XU}^{3}$ \\ ${ }^{1}$ Department of Ophthalmology, The Central Hospital of Wuhan, Wuhan, Hubei 430014; ${ }^{2}$ Department of Gastroenterology, \\ Tongji Hospital, Tongji Medical College, Huazhong University of Science and Technology, Wuhan, Hubei 430030; \\ ${ }^{3}$ Department of Ophthalmology, Shanghai First Hospital of Shanghai Jiao Tong University \\ School of Medicine, Shanghai 200080, P.R. China
}

Received June 23, 2015; Accepted January 21, 2016

DOI: $10.3892 /$ ijmm.2016.2478

\begin{abstract}
Ectopic apoptosis of vascular cells plays a critical role in the early stage development of diabetic retinopathy (DR). Uncoupling protein 2 (UCP2) is a mitochondrial modulator which protects against endothelial dysfunction. However, the role which UCP2 plays in endothelial apoptosis and its association with DR was unclear. In the present study, we investigated whether UCP2 functioned as an inhibitor of DR in endothelial cells. Firstly, we noted that in UCP2-knockout mice retinal cell death and damage in vivo was similar to that of $\mathrm{db} / \mathrm{db}$ diabetic mice. Additionally, UCP2 knockdown induced caspase-3 activation and exaggerated high glucose (HG)-induced apoptosis of human umbilical vein endothelial cells (HUVECs). Conversely, adenovirus-mediated UCP2 overexpression inhibited the apoptosis of HUVECs and HG-induced caspase-3 activation. Furthermore, HG treatment resulted in the opening of the permeability transition pore (PTP) and liberation of cytochrome $c$ from mitochondria to the cytosol in HUVECs. Notably, UCP2 overexpression inhibited these processes. Furthermore, adenovirus-mediated UCP2 overexpression led to a significant increase in intracellular nitric oxide (NO) levels and a decrease in reactive oxygen species (ROS) generation in HUVECs. Collectively, these data suggest that UCP2 plays an
\end{abstract}

Correspondence to: Dr Xunan Fu, Department of Ophthalmology, The Central Hospital of Wuhan, Wuhan, Hubei 430014, P.R. China E-mail: fxayanke@163.com

Dr Xun Xu, Department of Ophthalmology, Shanghai First Hospital of Shanghai Jiao Tong University School of Medicine, Shanghai 200080, P.R. China

E-mail: drxuxun@sjtu.edu.cn

*Contributed equally

Key words: uncoupling protein 2, diabetic retinopathy, apoptosis, endothelial cells anti-apoptotic role in endothelial cells. Thus, we suggest that approaches which augment UCP2 expression in vascular endothelial cells aid in preventing the early stage development and progression of DR.

\section{Introduction}

Diabetic retinopathy (DR) is the most common complication of diabetes and the principal cause of blindness in working-age individuals (1). The early stages of DR are characterized by microvascular cell damage (2). Microvascular cell damage is associated with thickening of the capillary endothelial basement membrane (BM) and pericyte apoptosis induced by hyperglycemia (3). In cases of DR, apoptotic cells have been found in all retinal layers, including retinal endothelial cells (4).

Previous investigations into the molecular mechanisms that cause DR have largely focused on vascular endothelial growth factor (VEGF) $(5,6)$. This may be attributed partly to the fact that the prominent clinical characteristics of DR have led to the general inference that DR is entirely of a microvascular nature. Although significant effort has been invested in elucidating the mechanisms that govern destructive preretinal neovascularization in DR (7), considerably less is known about the cellular processes that lead to increased retinal vascular apoptosis. Mitochondrial uncoupling protein 2 (UCP2) is a novel member of the mitochondrial anion carrier family, and displays $60 \%$ sequence identity with the well-known thermogenic UCP1 from brown adipose tissue (8). Previous studies have suggested that UCP2 is involved in the control of mitochondrial membrane potential (9) and the generation of reactive oxygen species (ROS) (10). Recently, a unifying hypothesis has emphasized the important role played by increased mitochondrial ROS production in complications of diabetes, including retinopathy (11). Previously, we demonstrated that peroxisome proliferator-activated receptor $\gamma$ (PPAR $\gamma)$-mediated changes to UCP2 involve the mitochondrial-ROS pathway, which is associated with a decreased VEGF-to-pigment epithelium-derived factor (PEDF) ratio caused by the effect that angiotensin-converting enzyme inhibitor (ACEI) exerts on DR (12). Moreover, we investigated the inhibition of high 
glucose-induced apoptosis by UCP2 in human umbilical vein endothelial cells (13). However, the more detailed mechanism of UCP2 in vascular endothelial cell apoptosis in DR has not been explored to date, to the best of our knowledge.

In the present study, using both UCP2-knockout mice and HUVECs, we provide evidence that UCP2 plays an anti-apoptotic role. UCP2-knockout mice exhibited retinal cell death and damage in vivo, which was similar to $\mathrm{db} / \mathrm{db}$ diabetic mice. Additionally, UCP2 knockdown exaggerated high glucose (HG)-induced apoptosis and caspase-3 activity in human umbilical vein endothelial cells (HUVECs). Furthermore, we demonstrated that adenovirus-mediated UCP2 overexpression exerted a protective effect on apoptosis, and investigated the opening of the permeability transition pore (PTP), cytochrome $c$ release, ROS generation and nitric oxide (NO) production in HUVECs. Thus, inducing UCP2 expression may represent an alternative therapeutic strategy in the early stage of DR.

\section{Materials and methods}

Cell culture and adenovirus transfection. HUVECs were obtained from ScienCell Research Laboratories (San Diego, CA, USA). The cells were cultured in endothelial cell medium (ECM) (ScienCell Research Laboratories) with 5\% (v/v) fetal bovine serum (FBS) at $37^{\circ} \mathrm{C}$ in an atmosphere with $5 \%(\mathrm{v} / \mathrm{v}) \mathrm{CO}_{2}$ and $95 \%$ humidity. When they reached confluence, the cells were maintained in $1 \%(\mathrm{v} / \mathrm{v})$ fetal calf serum and exposed to normal amounts of glucose $(5.5 \mathrm{mmol} / \mathrm{l})$ or high amounts of glucose (30 $\mathrm{mmol} / \mathrm{l}$ ) for 3-7 days, during which time the medium was changed every 2 days. When HUVECs reached approximately $50 \%$ confluence in fresh serum-free medium, they were transiently transduced with control adenovirus $\beta$-galactosidase (Ad- $\beta$-gal) or with adenovirus overexpressing UCP2 (Genechem, Shanghai, China) at multiplicities of infection (MOI) of 10. The cells were further cultured in ECM with $5 \%$ (v/v) FBS after infection for $4 \mathrm{~h}$ and then selected using $200 \mu \mathrm{m} / \mathrm{ml}$ puromycin (Thermo Fisher Scientific, Waltham, MA, USA). The stable overexpression lines were established when more than $95 \%$ of the transduced cells were found to strongly express green fluorescent protein (GFP) under a fluorescence microscope (BX51; Olympus, Tokyo, Japan).

Animals and sample preparation. All experiments in the present study comply with the requirements of the Association for Research in Vision and Ophthalmology Statement for the Use of Animals in Ophthalmic and Vision Research. Eighteen-week-old male C57 mice weighing $20 \mathrm{~g}$ and $\mathrm{db} / \mathrm{db}$ diabetic mice weighing $20 \mathrm{~g}$ were obtained from the Shanghai Laboratory Animal Center of Chinese Academy of Sciences (Jiuting, Shanghai, China). UCP2-deficient mice weighing $20 \mathrm{~g}$ were obtained from the Shanghai Biomodel Organism Research Center (Jiuting, Shanghai, China). This study was approved by the Ethics Committee of Shanghai Jiao Tong University School of Medicine.

UCP2 siRNA transfection. Target sequences were aligned to the human genome database in a BLAST search to ensure that the chosen sequences were not highly homologous with those of other genes. Cells were seeded in 6-well plates and cultured in drugfree medium (ScienCell Research Laboratories, Carlsbad, CA,
USA). When cells reached 90-95\% confluence they were washed twice with phosphate-buffered saline (PBS) and grown in $2 \mathrm{ml}$ ECM without antibiotics. Using Lipofectamine 2000 reagent (Invitrogen, Carlsbad, CA, USA), the indicated concentration (50 nM) of UCP2 siRNA oligo (Genechem) was transfected into HUVECs according to the manufacturer's instructions. Cells transfected with control siRNA served as a negative control. Forty-eight hours later, the expression levels of UCP2 were evaluated by western blot analysis. The cells transfected with siRNA were used for experiments $48 \mathrm{~h}$ after transfection.

Western blot analysis. Western blot analysis was performed, as previously described (14). Briefly, the primary antibodies used to probe the membranes included anti-UCP2 (1:500, cat. no. ab67241; Abcam, Cambridge, UK), anti-cytochrome $c$ (1:200, cat. no. 1896-1; Epitomics, Burlingame, CA, USA), anti-caspase-3 (1:5,000, cat. no. ab32351; Abcam) and anti$\beta$-actin (1:1,000, cat. no. A1978; Sigma-Aldrich, St. Louis, MO, USA). Experiments were repeated in triplicate.

Assessment of the release of cytochrome $c$ and subcellular localization of UCP2 protein expression. For the analysis of the release of cytochrome $c$ from mitochondria into the cytosol, proteins in the cytosol and mitochondrial fractions were separated by SDS/PAGE. Bands of proteins were transferred onto a PVDF membrane (Millipore, Bedford, MA, USA). Cytochrome $c$ was detected with an enhanced chemiluminescence western blot analysis system (Amersham, Buckinghamshire, UK) with monoclonal antibodies specific to cytochrome $c$ (1:200, cat. no. 1896-1; Epitomics). The same method for western blot analyses was used on cytosolic and mitochondrial preparations using antibody against human UCP2 (1:500, cat. no. ab67241; Abcam) and subunit IV of cytochrome oxidase (1:500, cat. no. ab110272; Abcam).

Flow cytometric analysis. For quantification of cellular viability, cells were double-stained with Annexin V and propidium iodide (PI) according to the manufacturer's instructions [Annexin V-fluorescein isothiocyanate (FITC) apoptosis detection kit (BD Biosciences, Franklin Lakes, NJ, USA)]. The proportion of apoptotic and necrotic cells was determined using FACSCalibur (Becton-Dickinson, San Jose, CA, USA). Annexin-V is a marker of apoptosis, and PI reflects the integrity of the cell membrane, thereby serving as a marker of necrosis.

Caspase-3 activity. Caspase-3 activity was determined using a caspase-3 colorimetric protease assay kit (Beyotime Institute of Biotechnology, Shanghai, China) following the manufacturer's instructions. Briefly, the kit uses spectrophotometry to detect the chromophore p-nitroaniline (pNA) after cleavage from the labeled substrate DEVD-pNA (DEVD is the sequence recognized by caspases). The free pNA was quantified using a spectrophotometer or a microtiter plate reader at $405 \mathrm{~nm}$. The ratio of absorbance from a sample to that from a control allows for the determination of the fold increase of caspase- 3 activity.

Assessment of mitochondrial permeability transition pore $(m P T P)$ opening. To determine the effect of UCP2 overexpression on mPTP opening, a previously validated cell model of mPTP opening was used (15). A microplate spectrofluorometer 
(SPECTRAmax GEM-INI-XS; Molecular Devices, Sunnyvale, CA, USA) was used to study stimulation of the fluorophore, tetramethylrhodamine methyl ester (TMRM), which accumulates in mitochondria, and generation of ROS within mitochondria. Culture medium was removed and replaced with Krebs imaging buffer. Cells were then loaded with $3 \mathrm{mM}$ TMRM for $15 \mathrm{~min}$ at $37^{\circ} \mathrm{C}$ and washed with Krebs imaging buffer (Guidechem, Shanghai, China). The time taken to induce mitochondrial membrane depolarization is recorded as a measurement of mPTP opening. This was defined as the time taken to reach half the maximum TMRM fluorescence intensity. Twenty transfected cells were randomly selected for the induction and detection of MPTP opening from each treatment group, and this was repeated in four independent experiments, providing a total of 80 cells/treatment group. As a positive control and in order to confirm that mitochondrial membrane depolarization was indicative of mPTP opening, following TMRM loading, a group of cells were pretreated for 10 min with the $\mathrm{mPTP}$ inhibitor, cyclosporin A (CsA; $0.2 \mathrm{mM}$; Selleck Chemicals, Houston, TX, USA), as previously described (16-18). The time taken to induce mPTP opening was recorded.

Measurement of intracellular ROS production and NO levels. In the present study, HUVECs were incubated with $10 \mu \mathrm{mol} / \mathrm{ml}$ carboxydichlorodihydrofluorescein diacetate (DCFH2-DA; Molecular Probes, Inc., Eugene, USA) at $37^{\circ} \mathrm{C}$. After $15 \mathrm{~min}$ incubation, the increase in DCFH2 oxidation was measured using a FACSCalibur (Becton-Dickinson) $(19,20)$. The NO level in HUVECs was measured in situ using DAF-FM diacetate (Sigma-Aldrich), as previously described (21).

Terminal deoxynucleotidyl transferase-mediated dUTP nick-end labeling (TUNEL) assay. Mice were sacrificed by cervical dislocation. Eyes were removed from four 8 week-old $\mathrm{db} / \mathrm{db}$ mice, four age-matched UCP2-KO mice and four age-matched normal mice were used as control retinas. The eyes were immediately enucleated and the retina was separated. The retina from one of the eyes was frozen in liquid nitrogen and stored at $-80^{\circ} \mathrm{C}$. TUNEL was performed on frozen sections using the DeadEnd ${ }^{\mathrm{TM}}$ TUNEL assay kit (Promega, Madison, WI, USA) and were counterstained with PI, according to the manufacturer's suggestions. Briefly, sections were hydrated with alcohol (100, 95 and 70\%), and then fixed in 3.7\% paraformaldehyde. After washing, the slides were incubated in a mixture of TdT, $\mathrm{Mn}^{2+}$, and TdT dNTP (Sangon Biotech, Shanghai, China) for $1 \mathrm{~h}$ at $37^{\circ} \mathrm{C}$. The reaction was stopped with TdT Stop Buffer (Trevigen, Gaithersburg, MD, USA) for $5 \mathrm{~min}$. After washing with deionized water, the slides were incubated with streptavidin-FITC (diluted 1:200) solution for $20 \mathrm{~min}$ at room temperature. Slides were counterstained, mounted, covered with coverslips, and visualized by confocal microscopy (LSM 510; Carl Zeiss, Inc., Oberkochen, Germany). Apoptotic cells were identified as doubly labeled with TdT fluorescein and PI, and only the nuclei which were clearly labeled yellow were scored.

Transmission electron microscopy. Tissue processing, electron microscopy, morphometric measurements [retinal capillary basement membrane thickness (BMT)], and statistical analysis were performed as detailed in our previous study (12). Eyes were removed from four 8 week-old $\mathrm{db} / \mathrm{db}$ mice, four age- matched UCP2-KO mice and four age-matched normal mice were used as control. Briefly, enucleated eyes were fixed in $2.5 \%(\mathrm{w} / \mathrm{v})$ glutaraldehyde in $0.1 \mathrm{M}$ cacodylate buffer $(\mathrm{pH} 7.4)$ containing $0.2 \%(\mathrm{v} / \mathrm{v})$ tannic acid, washed in the same buffer, and post-fixed in $0.5 \%(\mathrm{v} / \mathrm{v})$ osmium tetroxide. Tissue sections were block stained with uranyl acetate, lead stained, dehydrated through a graded series of ethanol, and embedded in Epon. One-micrometer-thick sections were examined with a JEM-1200EX transmission electron microscope (JEOL, Ltd., Akishima, Japan). Computer-assisted morphometric measurements (The Image Center of Beijing University of Aeronautics and Astronautics, Beijing, China) were performed on electron micrographs taken from 12 randomly selected capillaries of the outer plexiform layer from four different tissue blocks of the same retina. Only cross-sectioned capillaries were considered. A total of 96 capillaries were evaluated in each experimental group.

Statistical analysis. SPSS 17.0 was used to analyze the experimental data. All experimental data are represented as the means \pm standard deviation (SD). One-way analysis of variance (ANOVA) followed by Student-Newman-Keuls test was used to compare the effect of treatment on the various parameters. Non-parametric data was analyzed using the Chi-square test or Fisher's exact method. A P-value $<0.05$ was considered to indicate a statistically significant difference.

\section{Results}

Retinal cell death and damage in mice. Loss of cells in the ganglion cell layer was due to apoptosis, and this was reflected as an increase in TUNEL in the ganglion cell layer of both the UCP2-knockout mice and db/db mice. We performed TUNEL on retinal sections to measure cell death in the UCP2-knockout mice and $\mathrm{db} / \mathrm{db}$ mice. Notably, we found that UCP2-knockout mice exhibited a DR-like phenotype, similar to $\mathrm{db} / \mathrm{db}$ mice. Fig. 1A and $\mathrm{C}$ illustrate increased TUNEL in the ganglion cell layer of UCP2-knockout mice and db/db mice compared to their normal counterparts. Furthermore, compared to the non-diabetic mice, the BMT was significantly increased in the UCP2-knockout mice and db/db mice $(\mathrm{P}<0.01)$ (Fig. 1B and D). However, there was no significant difference in BMT between UCP2-knockout mice and $\mathrm{db} / \mathrm{db}$ mice. Therefore, these results demonstrate that knockdown of UCP2 is a critical factor for retinal cell death and damage.

Knockdown of UCP2 exaggerates HG-induced apoptosis and activated caspase-3 in HUVECs. UCP2 siRNA effectively reduced endogenous levels of UCP2 protein compared to control siRNA (Fig. 2A). UCP2 siRNA increased caspase-3 expression to a significant extent, whereas control siRNA did not (Fig. 2B). UCP2 siRNA exaggerated HG-induced apoptosis compared to control-siRNA infected cells (Fig. 2C and D). These data demonstrate that endogenous UCP2 expression is important for preventing apoptosis in HUVECs.

Subcellular localization of UCP2 protein in UCP2-overexpressing HUVECs. In the present study, HUVECs were infected with adenovirus carrying UCP 2 cDNA in sense orientation at an MOI of 1,5 and $10 \mathrm{pfu} / \mathrm{cell}$ for $24 \mathrm{~h}$. Immunofluorescence micros- 

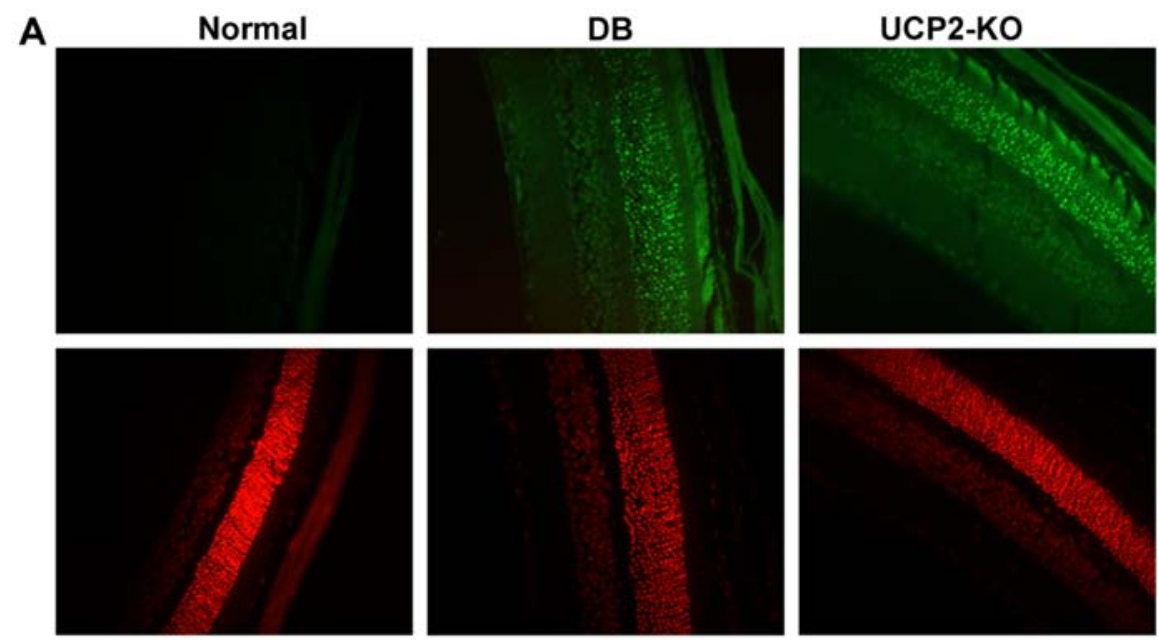

B
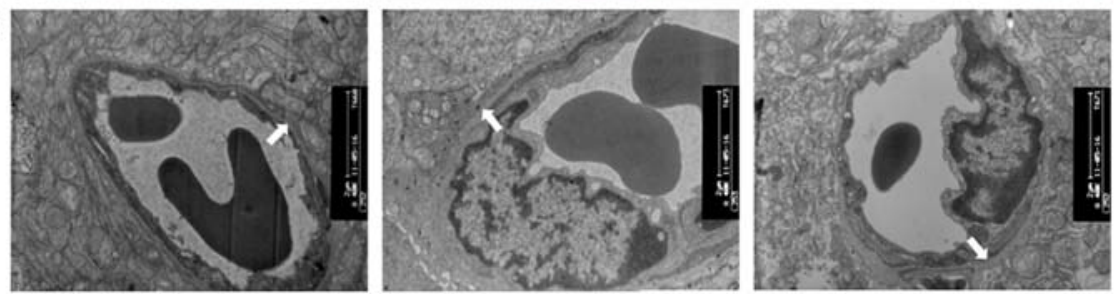

C

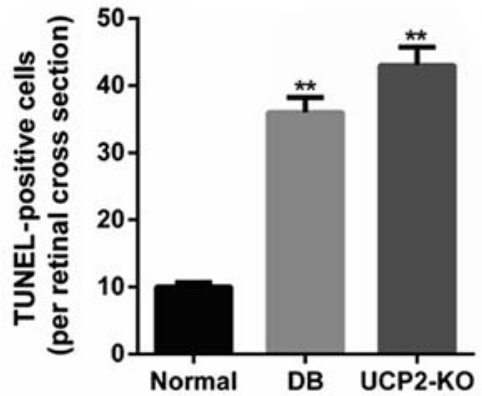

D

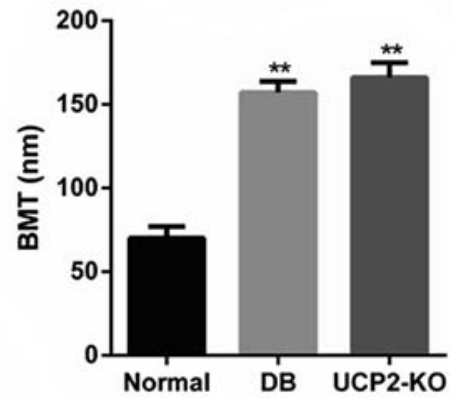

Figure 1. Effect of uncoupling protein 2 (UCP2) on retinal cell death and damage in UCP2-knockout (KO) mice. (A) Retinal distribution of TUNEL-positive cells in normal, db/db, and UCP2-KO mice. Sections were counterstained with PI. For clearer TUNEL identification, the retinal area of the diabetic animal was studied using separated and merged TUNEL and PI staining. (B) Transmission electron microscopy of a capillary from the outer plexiform layer of the mouse retina in the three groups. Arrows denote the segment of the outer capillary BM between the endothelial cells and glia limitans, which was used to measure basement membrane width (original magnification, x9,000). (C) Retinal quantitation of TUNEL-positive cells. TUNEL-positive cells were counted in 10 adjacent locations along the vertical meridian within $4 \mathrm{~mm}$ of the optic disk (10 fields/retina section). (D) Retinal capillaries (basement membrane thickness, $\mathrm{nm}$ ) in the three groups. Data are represented as the means \pm SD from eight mice per group, and the experiments were repeated independently at least three times with similar results. Normal, C57 mice. ${ }^{* *} \mathrm{P}<0.01 \mathrm{vs}$. control.

copy was then used in order to observe UCP2 eGFP expression in infected cells. At an MOI of $10 \mathrm{pfu} / \mathrm{cell}$, UCP2 eGFP expression was detected in $>95 \%$ of cultured cells (Fig. 3A). UCP2 was overexpressed mainly within the mitochondria (Fig. 3B and C).

UCP2 overexpression inhibits $H G$-induced apoptosis and caspase-3 activity in HUVECs. HG increased HUVEC apoptosis by 4.1-fold; UCP2 overexpression reduced HG-induced apoptosis by $65 \%$ in HUVECs (Fig. 4A). FACScan analysis revealed that UCP2 overexpression inhibited both $\mathrm{HG}$-induced apoptosis and necrosis of HUVECs (Fig. 4A and B). HG induced a significant increase in caspase- 3 activity in HUVECs (Fig. 4C and D). The HG-induced increase in caspase-3 activity was inhibited by transfection with Ad-UCP2. The generation of active caspase- 3 from the proenzyme was enhanced in the presence of HG (Fig. 4C and D). HG-induced cleavage of caspase-3 into active subunits was thus prevented in the presence of $\mathrm{UCP} 2$.

UCP2 overexpression delays MPTP opening and blocks cytochrome c release. UCP2 overexpression in HUVECs decreased the susceptibility of the cells to MPTP opening. UCP2 overexpression in HUVECs delayed the time of mPTP opening by $1.5 \pm 0.2$-fold when compared to control values $(\mathrm{P}<0.01)$ (Fig. 5A). In addition, treatment with $\mathrm{HG}$ resulted in the liberation of cytochrome $c$ from mitochondria to the cytosol (Fig. 5B). The liberation of cytochrome $c$ by HG was blocked in cells overexpressing UCP2 (Fig. 5B).

UCP2 overexpression suppresses intracellular ROS production and increases the intracellular NO levels in HUVECs. Previous research has indicated that UCP2 is activated by 
A

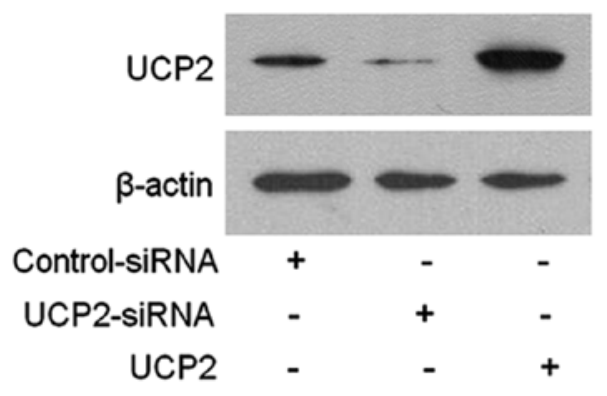

C
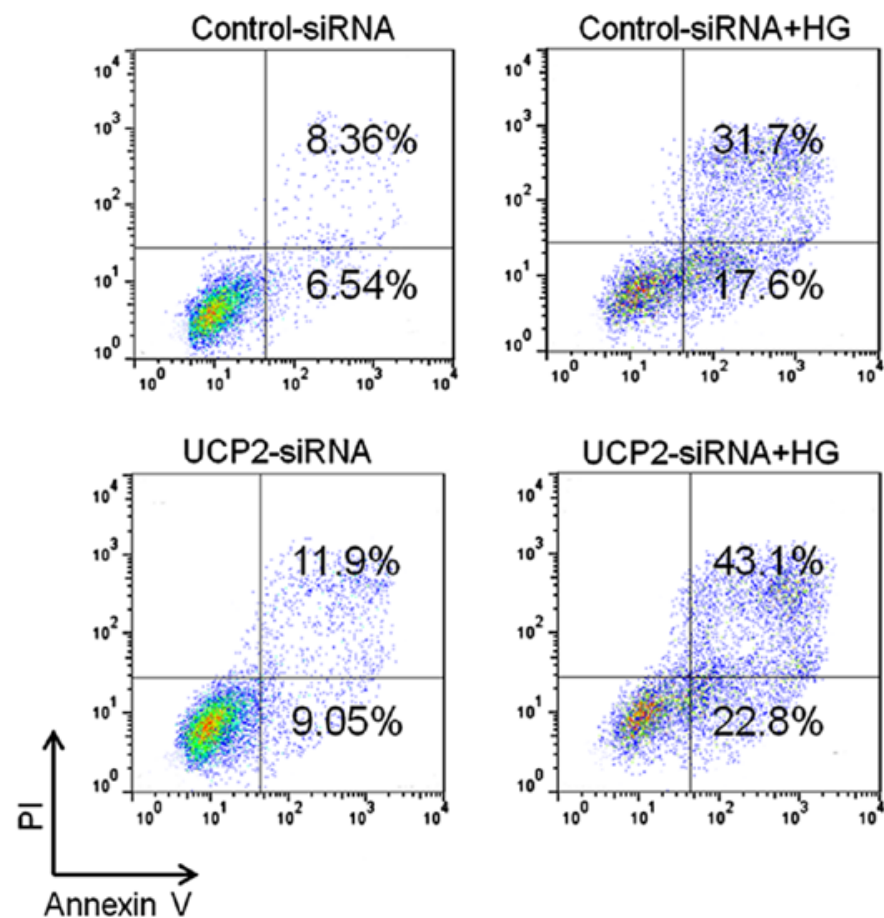

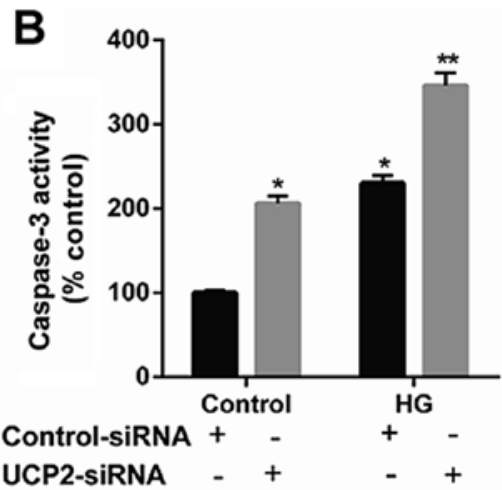

D
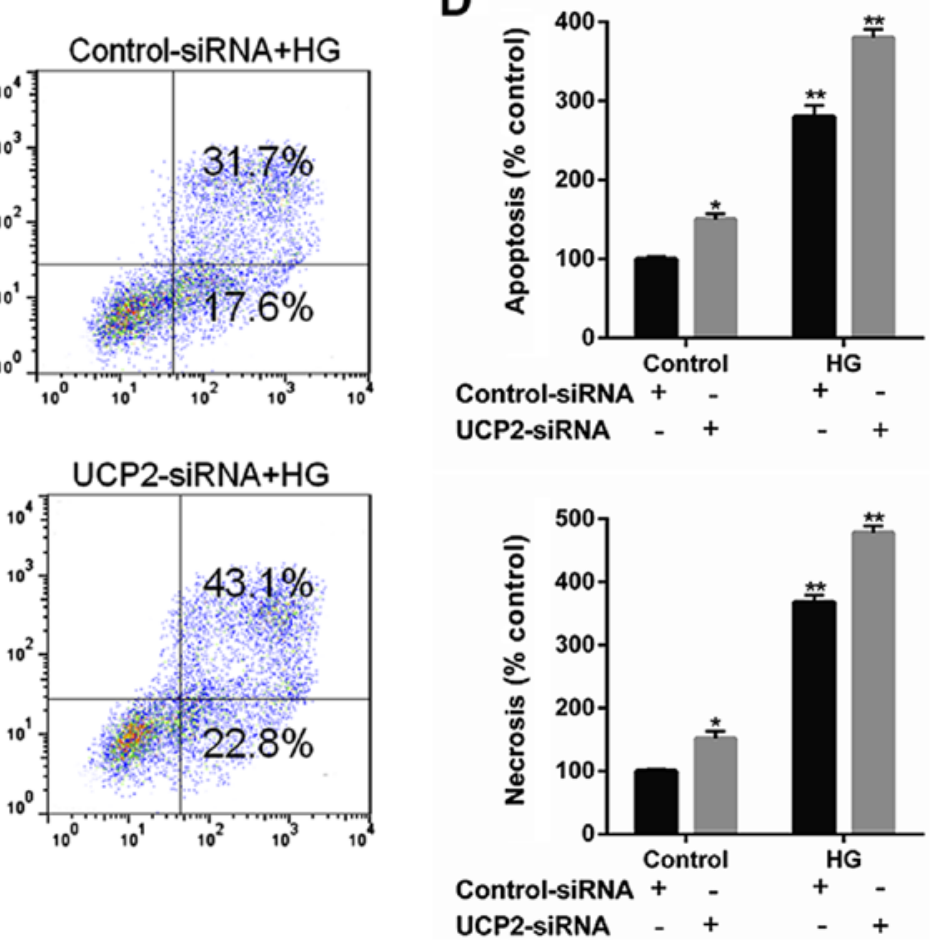

Figure 2. Effect of uncoupling protein 2 (UCP2)-knockdown on high glucose (HG)-induced apoptosis and caspase-3 activation in human umbilical vein endothelial cells (HUVECs). (A) Two days after transfection, UCP2 protein expression was examined by western blot analysis. (B) Transfected cells were maintained in media containing $0.5 \%$ (v/v) FBS for $12 \mathrm{~h}$, and $30 \mathrm{M} \mathrm{HG}$ for $16 \mathrm{~h}$. Following treatment, caspase-3 activation and apoptosis were analyzed using a caspase fluorescence assay kit. (C) Transfected cells treated with HG were double stained with Annexin V and PI using the Annexin V-FITC apoptosis detection kit I. The proportion of apoptotic and necrotic cells was determined by FACSCalibur. (D) Effect of UCP overexpression on HG-induced apoptosis and necrosis. Apoptotic and necrotic cells were identified by staining with Annexin V (early apoptosis) or Annexin V/PI (late apoptosis) and PI, respectively. ${ }^{*} \mathrm{P}<0.01$ vs. control-siRNA and ${ }^{* *} \mathrm{P}<0.01$ vs. Control-UCP2-siRNA.

ROS and attenuates excessive ROS production in the form of a negative feedback mechanism (22-24). Thus, in the present study, the levels of ROS were determined in HUVECs. Incubation with HG significantly increased intracellular ROS generation. However, upon UCP2 overexpression, we noted that the increase in ROS production effected by HG was suppressed (Fig. 6A).

It has previously been noted that NO produced by eNOS exerts anti-inflammatory effects on the vascular wall, and inhibits the migration and proliferation of vascular smooth muscle cells (25). Thus, in the present study we detected the intracellular NO level in HUVECs. We noted that intracellular NO levels were decreased by HG, whereas UCP2 overexpression increased intracellular NO levels (Fig. 6B).

\section{Discussion}

Therapeutic strategies to treat complications associated with DR previously consisted predominantly of controlling systemic vascular deregulation (26-29). Although laser photocoagulation and targeted treatments, such as locally administered corticosteroids, are currently available, their off-target effects underscore the need to explore novel therapeutic approaches $(30,31)$. In the present study, we provide evidence that UCP2 plays a critical role in vascular cell apoptosis induced by HG in DR.

A previous study has shown that loss of retinal capillary endothelial and mural cells and focal BM thickening has been noted in streptozotocin-induced diabetic rats (32). Similarly, in the present study we performed TUNEL assay on retinal 
A
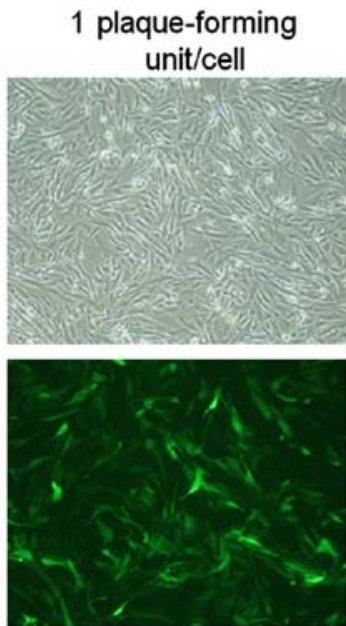

B

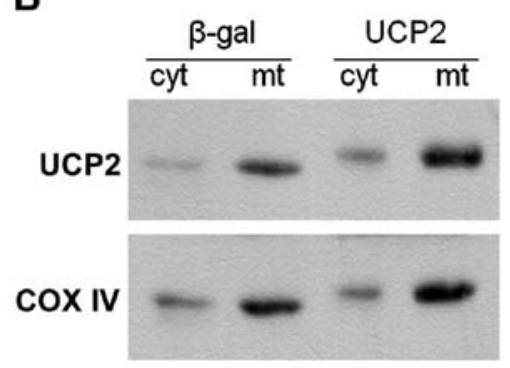

5 plaque-forming units/cell
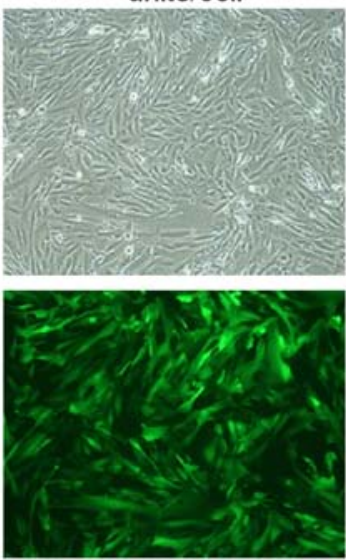

C

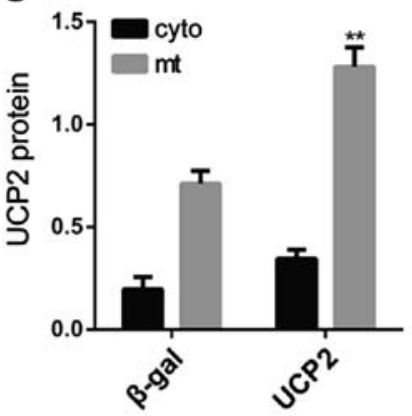

10 plaque-forming units/cell
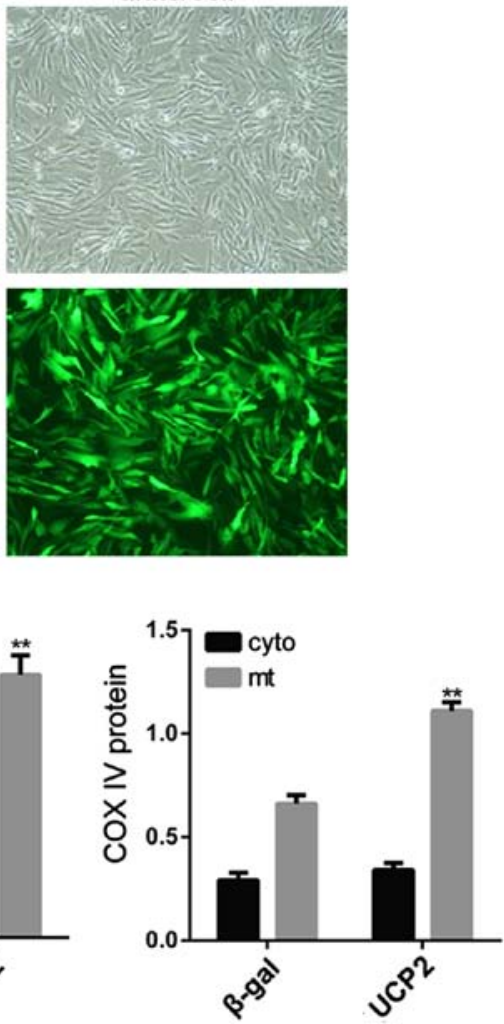

Figure 3. Subcellular localization of uncoupling protein 2 (UCP2) protein in UCP2-overexpressing human umbilical vein endothelial cells (HUVECs). (A) Overexpression of UCP2 in HUVECs by transient infection with recombinant adenovirus constructs containing UCP2 sense cDNA and eGFP (Ad-UCP2-eGFP). Live cell images were taken under an inverted epifluorescence microscope, showing green fluorescence for overexpressed eGFP-UCP2 for the different concentrations of adenovirus-UCP2-eGFP (MOI of 1, 5 and $10 \mathrm{pfu} / \mathrm{cell}$, respectively). (B) Adenovirus-mediated gene transfer (6x10 6 pfu/ml) to confluent HUVECs was performed by 1-h infection at $37^{\circ} \mathrm{C}$ in ECM without serum. After $48 \mathrm{~h}$, western blot analysis was performed on cytosolic and mitochondrial preparations using an antibody against human UCP2 and subunit IV of cytochrome oxidase, a standard mitochondrial marker. UCP 2 was overexpressed mainly within mitochondria. (C) UCP2 and COX IV protein levels were analyzed. Cyt/cyto, cytosol; mt, mitochondria. The values are presented as the means $\pm \mathrm{SD}$ of three independent experiments. ${ }^{* *} \mathrm{P}<0.01$ vs. $\mathrm{mt}+\beta$-gal.

A
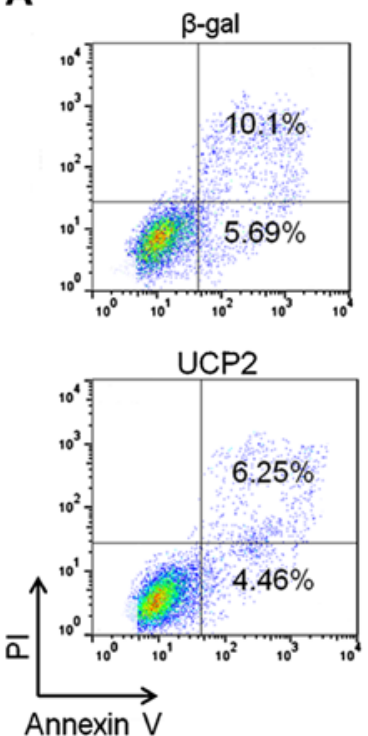

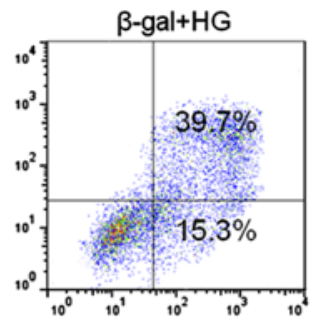

B

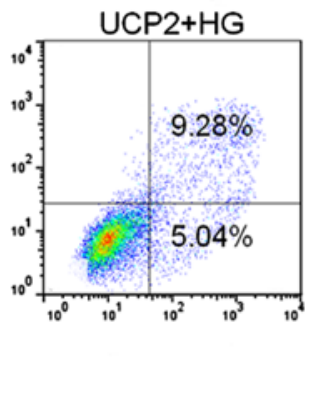

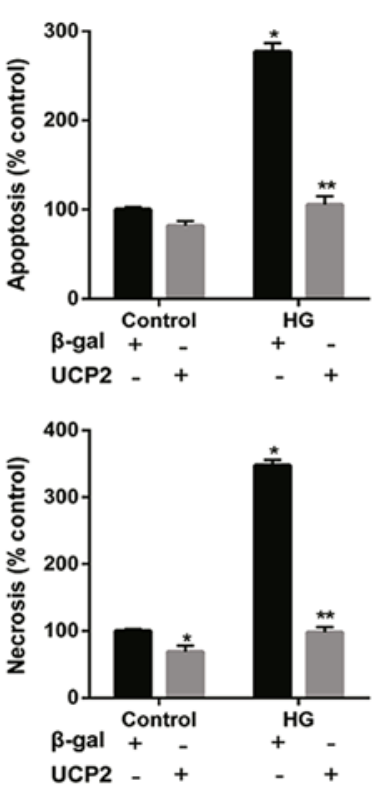

C

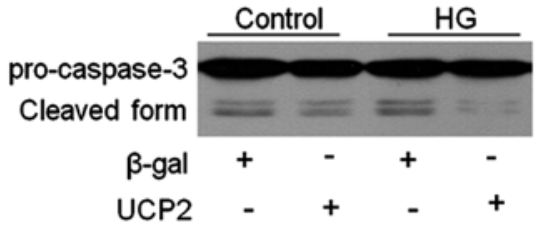

D

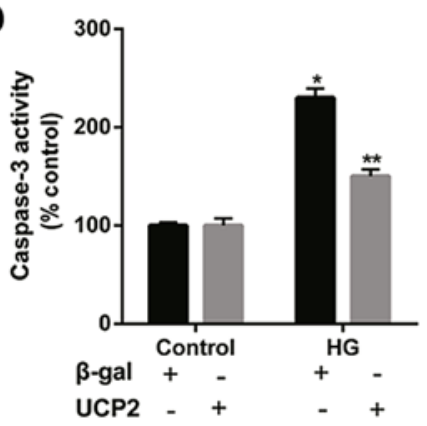

Figure 4. Effect of uncoupling protein 2 (UCP2) overexpression on high glucose (HG)-induced apoptosis and caspase activation in human umbilical vein endothelial cells (HUVECs). (A) Ad-UCP2 or adenovirus $\beta$-galactosidase (Ad- $\beta$-gal)-infected HUVECs treated with HG were double stained with Annexin V and PI using the Annexin V-FITC apoptosis detection kit. The proportion of apoptotic and necrotic cells was determined by FACSCalibur. (B) Effect of UCP2 overexpression on HG-induced apoptosis and necrosis. Apoptotic and necrotic cells were identified by staining with Annexin V (early apoptosis) or Annexin V/PI (late apoptosis) and PI, respectively. (C) Western blot analysis for the active subunit of caspase-3. (D) Caspase-3 activation was analyzed by measuring the activity using a caspase- 3 colorimetric protease assay kit. The values are presented as the means \pm SD of three independent experiments. ${ }^{*} \mathrm{P}<0.01$ vs. control and ${ }^{* *} \mathrm{P}<0.01$ vs. $\beta$-gal. 
B

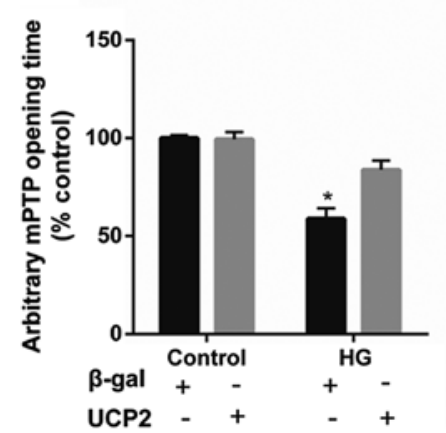

Figure 5. Effect of uncoupling protein 2 (UCP2) overexpression on permeability transition pore (PTP) opening and cytochrome $c$ release. (A) Overexpression of UCP2 in human umbilical vein endothelial cells (HUVECs) significantly delayed the time of mPTP opening compared to the adenovirus $\beta$-galactosidase (Ad- $\beta$-gal) control. (B) Western blot analysis of the release of cytochrome $c$ from mitochondria into the cytosol. The values are presented as the means $\pm \mathrm{SD}$ of three independent experiments. ${ }^{*} \mathrm{P}<0.01$ vs. control.

A

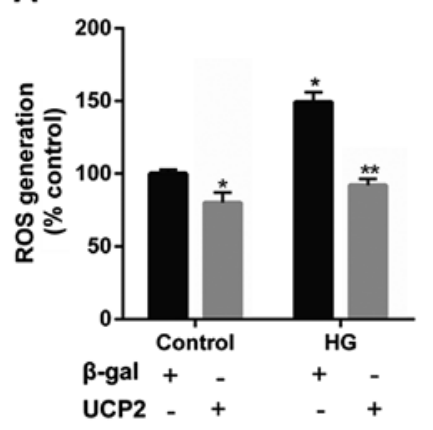

B

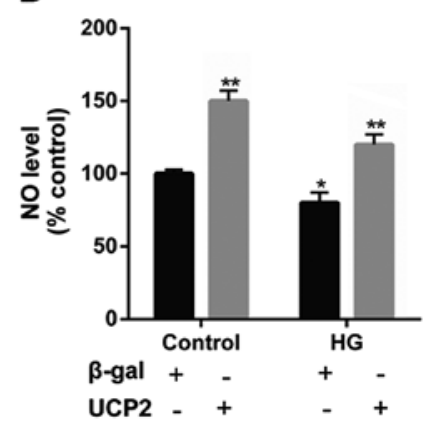

Figure 6. Effect of uncoupling protein 2 (UCP2) overexpression on reactive oxygen species (ROS) generation and NO production in human umbilical vein endothelial cells (HUVECs). Quiescent HUVECs were infected with Ad-UCP2 or Ad- $\beta$-gal. Two days after infection, cells were maintained in media containing $0.5 \%$ (v/v) FBS for $12 \mathrm{~h}$, and exposed to $30 \mathrm{M}$ high glucose (HG) for $6 \mathrm{~h}$. (A) After $15 \mathrm{~min}$ incubation with $10 \mu \mathrm{mol} / \mathrm{ml}$ DCFH2-DA, the increase in DCFH2 oxidation was measured using FACSCalibur. (B) NO levels in HUVECs were measured in situ using DAF-FM diacetate. Values are presented as the means $\pm \mathrm{SD}$ of three independent experiments. ${ }^{*} \mathrm{P}<0.01$ vs. control and ${ }^{* *} \mathrm{P}<0.01$ vs. $\beta$-gal.

sections to measure cell death in the UCP2-knockout mice, $\mathrm{db} / \mathrm{db}$ mice and C57 mice. Notably, UCP2-knockout mice exhibited DR-like damage, similar to $\mathrm{db} / \mathrm{db}$ mice. We noted an increase in TUNEL in the ganglion cell layer of UCP2-knockout mice and $\mathrm{db} / \mathrm{db}$ mice compared to the wild-type mice. Furthermore, compared with non-diabetic mice, the BMT was also significantly increased in the UCP2-knockout mice and db/db mice. There was no significant difference between UCP2-knockout mice and $\mathrm{db} / \mathrm{db}$ mice. These results suggest that UCP2 exerts a protective effect in the early stages of DR.

Endothelial apoptosis is an important early event in the pathogenesis of DR, and increased intracellular oxidative stress plays an important causative role in the pathogenesis of endothelial apoptosis (33-36). Moreover, caspase-3 activation is a crucial step in the process of apoptosis. Activation of caspase-3 is essential for cell execution and occurs immediately prior to the development of morphological changes consistent

with apoptosis (37). Hence, a cell with activated caspase-3 is considered destined to die. In agreement with previous research on other cell types (38), in the present study, we noted that suppression of UCP2 expression using UCP2-specific siRNA exaggerated caspase-3 activation and apoptosis induced by HG. Conversely, overexpression of UCP2 inhibited HG-induced endothelial apoptosis and caspase-3 activation. In addition, UCP2 overexpression decreased ROS generation, PTP opening, and cytochrome $c$ release induced by HG. These data demonstrate that endogenous UCP2 expression is important for preventing apoptosis in HUVECs.

The endothelium acts not only as a barrier, but also as a regulator of vascular tone and smooth muscle cell growth, migration, and proliferation. Vascular tone is modulated through the release of relaxing and contracting substrates. Of these, NO is physiologically the most important regulator of vascular tone. Although a previous study demonstrated that the vascular benefit of UCP2 was due to increased NO bioavailability after the inhibition of ROS production in HG-treated primary mouse aortic endothelial cells (39), in the present study we showed that HG significantly increased intracellular ROS production and decreased intracellular NO levels in HUVECs. UCP2 overexpression reversed these effects. Thus, we suggest that UCP2 reduces ROS production and increases NO levels.

\section{Acknowledgements}

The present study was supported by grants from the Research Fund for the National Nature Science Funding of China (nos. 30930097, 81273424 and 81170862), the Major National Science and Technology Projects during the 12th Five-Year Plan (no. 011ZX09302-007-02).

\section{References}

1. Cerani A, Tetreault N, Menard C, Lapalme E, Patel C, Sitaras N, Beaudoin F, Leboeuf D, De Guire V, Binet F, et al: Neuron-derived semaphorin $3 \mathrm{~A}$ is an early inducer of vascular permeability in diabetic retinopathy via neuropilin-1. Cell Metab 18: 505-518, 2013.

2. Barot M, Gokulgandhi MR, Patel S and Mitra AK: Microvascular complications and diabetic retinopathy: recent advances and future implications. Future Med Chem 5: 301-314, 2013.

3. Geraldes P, Hiraoka-Yamamoto J, Matsumoto M, Clermont A, Leitges M, Marette A, Aiello LP, Kern TS and King GL: Activation of PKC-delta and SHP-1 by hyperglycemia causes vascular cell apoptosis and diabetic retinopathy. Nat Med 15: 1298-1306, 2009.

4. Jiang Y, Zhang Q, Soderland C and Steinle JJ: TNF $\alpha$ and SOCS3 regulate IRS-1 to increase retinal endothelial cell apoptosis. Cell Signal 24: 1086-1092, 2012.

5. Montero JA, Ruiz-Moreno JM and Correa ME: Intravitreal anti-VEGF drugs as adjuvant therapy in diabetic retinopathy surgery. Curr Diabetes Rev 7: 176-184, 2011.

6. Hammes HP, Feng Y, Pfister F and Brownlee M: Diabetic retinopathy: targeting vasoregression. Diabetes 60: 9-16, 2011.

7. Bressler NM, Beck RW and Ferris FL III: Panretinal photocoagulation for proliferative diabetic retinopathy. N Engl J Med 365: 1520-1526, 2011

8. Zhang CY,Parton LE, Ye CP, Krauss S, Shen R, Lin CT, Porco JA Jr and Lowell BB: Genipin inhibits UCP2-mediated proton leak and acutely reverses obesity- and high glucose-induced beta cell dysfunction in isolated pancreatic islets. Cell Metab 3: 417-427, 2006.

9. Brand MD and Esteves TC: Physiological functions of the mitochondrial uncoupling proteins UCP2 and UCP3. Cell Metab 2: 85-93, 2005.

10. Bouillaud F: UCP2, not a physiologically relevant uncoupler but a glucose sparing switch impacting ROS production and glucose sensing. Biochim Biophys Acta 1787: 377-383, 2009. 
11. Wilkinson-Berka JL, Rana I, Armani R and Agrotis A: Reactive oxygen species, Nox and angiotensin II in angiogenesis: implications for retinopathy. Clin Sci (Lond) 124: 597-615, 2013.

12. Zheng Z, Chen H, Ke G, Fan Y, Zou H, Sun X, Gu Q, Xu X and Ho PC: Protective effect of perindopril on diabetic retinopathy is associated with decreased vascular endothelial growth factor-to-pigment epithelium-derived factor ratio: involvement of a mitochondria-reactive oxygen species pathway. Diabetes 58 : 954-964, 2009

13. He Y, Wang $\mathrm{N}$, Shen $\mathrm{Y}$, Zheng $\mathrm{Z}$ and $\mathrm{Xu} \mathrm{X}$ : Inhibition of high glucose-induced apoptosis by UCP2 in human umbilical vein endothelial cells. Int J Mol Med 33: 1275-1281, 2014.

14. Luan Z, He Y, Alattar M, Chen Z and He F: Targeting the prohibitin scaffold-CRAF kinase interaction in RAS-ERK-driven pancreatic ductal adenocarcinoma. Mol Cancer 13: 38, 2014.

15. Zorov DB, Filburn CR, Klotz LO, Zweier JL and Sollott SJ: Reactive oxygen species (ROS)-induced ROS release: a new phenomenon accompanying induction of the mitochondrial permeability transition in cardiac myocytes. J Exp Med 192 1001-1014, 2000.

16. Hausenloy DJ and Yellon DM: The mitochondrial permeability transition pore: its fundamental role in mediating cell death during ischaemia and reperfusion. J Mol Cell Cardiol 35: 339-341, 2003

17. Davidson SM, Hausenloy D, Duchen MR and Yellon DM Signalling via the reperfusion injury signalling kinase (RISK) pathway links closure of the mitochondrial permeability transition pore to cardioprotection. Int J Biochem Cell Biol 38 : 414-419, 2006

18. Lim SY, Davidson SM, Paramanathan AJ, Smith CC, Yellon DM and Hausenloy DJ: The novel adipocy tokine visfatin exerts direct cardioprotective effects. J Cell Mol Med 12: 1395-1403, 2008.

19. Bass DA, Parce JW, Dechatelet LR, Szejda P, Seeds MC and Thomas M: Flow cytometric studies of oxidative product formation by neutrophils: a graded response to membrane stimulation. J Immunol 130: 1910-1917, 1983.

20. van Reyk DM, King NJ, Dinauer MC and Hunt NH: The intracellular oxidation of $2^{\prime}, 7^{\prime}$-dichlorofluorescin in murine T lymphocytes. Free Radic Biol Med 30: 82-88, 2001

21. Shao C, Stewart V, Folkard M, Michael BD and Prise KM: Nitric oxide-mediated signaling in the bystander response of individually targeted glioma cells. Cancer Res 63: 8437-8442, 2003.

22. Jezek P and Garlid KD: Mammalian mitochondrial uncoupling proteins. Int J Biochem Cell Biol 30: 1163-1168, 1998.

23. Derdak Z, Mark NM, Beldi G, Robson SC, Wands JR and Baffy G: The mitochondrial uncoupling protein-2 promotes chemoresistance in cancer cells. Cancer Res 68: 2813-2819, 2008.

24. Baffy G: Uncoupling protein-2 and cancer. Mitochondrion 10: 243-252, 2010

25. Williams IL, Wheatcroft SB, Shah AM and Kearney MT: Obesity, atherosclerosis and the vascular endothelium: mechanisms of reduced nitric oxide bioavailability in obese humans. Int $\mathrm{J}$ Obes Relat Metab Disord 26: 754-764, 2002.
26. Bhattacharjee PS, Huq TS, Potter V, Young A, Davenport IR, Graves R, Mandal TK, Clement C, McFerrin HE, Muniruzzaman S, et al: High-glucose-induced endothelial cell injury is inhibited by a peptide derived from human apolipoprotein $\mathrm{E}$. PLoS One 7: e52152, 2012.

27. Titchenell PM and Antonetti DA: Using the past to inform the future: anti-VEGF therapy as a road map to develop novel therapies for diabetic retinopathy. Diabetes 62: 1808-1815, 2013.

28. Ababneh OH, Yousef YA, Gharaibeh AM, Abu Ameerh MA, Abu-Yaghi NE and Al Bdour MD: Intravitreal bevacizumab in the treatment of diabetic ocular neovascularization. Retina 33: 748-755, 2013.

29. Verma A, Shan Z, Lei B, Yuan L, Liu X, Nakagawa T, Grant MB, Lewin AS, Hauswirth WW, Raizada MK and Li Q: ACE2 and Ang-(1-7) confer protection against development of diabetic retinopathy. Mol Ther 20: 28-36, 2012.

30. Zhang Z, Meng X, Wu Z, Zou W, Zhang J, Zhu D, Chen T and Zhang Q: Changes in choroidal thickness after panretinal photocoagulation for diabetic retinopathy: a 12-week longitudinal study. Invest Ophthalmol Sci 56: 2631-2638, 2015.

31. Kumar B, Gupta SK, Saxena R and Srivastava S: Current trends in the pharmacotherapy of diabetic retinopathy. J Postgrad Med 58: 132-139, 2012.

32. Liu BC, Chen Q, Luo DD, Sun J, Phillips AO, Ruan XZ and Liu NF: Mechanisms of irbesartan in prevention of renal lesion in streptozotocin-induced diabetic rats. Acta Pharmacol Sin 24: 67-73, 2003.

33. Devi TS, Hosoya K, Terasaki T and Singh LP: Critical role of TXNIP in oxidative stress, DNA damage and retinal pericyte apoptosis under high glucose: implications for diabetic retinopathy. Exp Cell Res 319: 1001-1012, 2013.

34. Rask-Madsen C and King GL: Vascular complications of diabetes: mechanisms of injury and protective factors. Cell Metab 17: 20-33, 2013

35. Fu D, Wu M, Zhang J, Du M, Yang S, Hammad SM, Wilson K, Chen $\mathbf{J}$ and Lyons TJ: Mechanisms of modified LDL-induced pericyte loss and retinal injury in diabetic retinopathy. Diabetologia 55: 3128-3140, 2012.

36. Kowluru RA, Mohammad G, dos Santos JM and Zhong Q: Abrogation of MMP-9 gene protects against the development of retinopathy in diabetic mice by preventing mitochondrial damage. Diabetes 60: 3023-3033, 2011.

37. Tyas L, Brophy VA, Pope A, Rivett AJ and Tavaré JM: Rapid caspase-3 activation during apoptosis revealed using fluorescence-resonance energy transfer. EMBO Rep 1: 266-270, 2000.

38. Mattiasson G, Shamloo M, Gido G, Mathi K, Tomasevic G, Yi S, Warden CH, Castilho RF, Melcher T, Gonzalez-Zulueta M, et al: Uncoupling protein-2 prevents neuronal death and diminishes brain dysfunction after stroke and brain trauma. Nat Med 9: 1062-1068, 2003.

39. Tian XY, Wong WT, Xu A, Lu Y, Zhang Y, Wang L, Cheang WS, Wang Y, Yao X and Huang Y: Uncoupling protein-2 protects endothelial function in diet-induced obese mice. Circ Res 110: 1211-1216, 2012 